\title{
O uso da terapia fotodinâmica como método alternativo de tratamento da candidíase oral
}

\section{The use of photodynamic therapy as an alternative method of treating oral candidiasis}

\author{
Paulo de Souza Teodoro ${ }^{*}$, Hugo Victor dos Santos Fernandes ${ }^{2}$, Elvys da Cunha Sá ${ }^{3}$, Lorena \\ Alves Coutinho Pimentel ${ }^{4}$
}

${ }^{1}$ Acadêmico de Odontologia, Instituto Macapaense de Ensino Superior - IMMES. Macapá-AP Brasil. E-mail: pdteodoro@hotmail.com *Autor para correspondência.

${ }^{2}$ Acadêmico de Odontologia, Instituto Macapaense de Ensino Superior - IMMES. Macapá-AP Brasil. E-mail: hugo.victor.hv88@gmaill.com ${ }^{3}$ Estomatologista, Unidade de Alta Complexidade em Oncologia do Hospital de Clínicas Alberto Lima e docente do colegiado de odontologia do Instituto Macapaense de Ensino Superior-IMMES, Macapá-AP, E-mail: elvyscunha@hotmail.com

${ }^{4}$ Doutoranda em Clínicas Odontológicas com área de concentração em Endodontia, Faculdade São Leopoldo Mandic, Campinas-SP e docente do colegiado de odontologia do Instituto Macapaense de Ensino Superior-IMMES, E-mail: lorenaalvescoutinho@hotmail.com

\author{
Palavras-chave \\ Candidíase Oral \\ Candida \\ Terapia Fotodinâmica
}

\begin{abstract}
A candidíase oral é a mais relevante infeç̧ão fúngica encontrada na cavidade oral humana. Esta patologia é uma infecção oportunista, na qual o fungo deixa de atuar como comensal e torna-se patogênico quando o hospedeiro apresenta fatores suscetíveis. Devido à ocorrência de resistência aos antifúngicos, além dos seus efeitos colaterais e interações medicamentosas, é necessário que novas terapêuticas sejam utilizadas. E, a Terapia Fotodinâmica (PDT) é uma intervenção que tem se mostrado uma excelente opção. Por isso, este artigo tem como objetivo abordar o uso da PDT como monoterapia e em associação com a terapia convencional no tratamento da candidiase oral, indicando desta forma se esta nova modalidade terapêutica vem a ser uma técnica substituta ou auxiliar no tratamento da candidíase oral. Desta maneira, foi utilizada a metodologia revisão de literatura baseada em livros, artigos científicos indexados nas bases da Scielo, PubMed, Medline e Lilacs. Foram utilizados como Critérios de inclusão: livros e artigos publicados nos últimos 10 anos, relacionados à temática do artigo, disponibilizados na íntegra. Critérios de exclusão: trabalhos não disponíveis na íntegra e publicações em anais de eventos. Critérios de qualificação: a leitura do título e do resumo, onde 2 livros e 27 artigos atenderam os critérios da pesquisa. Os resultados demonstraram que apesar do uso da PDT de maneira isolada ter combatido a candidíase oral de maneira satisfatória, os melhores resultados foram obtidos na associação de terapias. Conclui-se que atualmente o melhor tratamento para a candidíase oral é a combinação da PDT com a os antifúngicos tradicionais.
\end{abstract}

\section{Keywords}

Oral Candidiasis

Candida

Photodynamic Therapy
Oral candidiasis is the most relevant fungal infection found in the human oral cavity. This condition is an opportunistic infection, in which the fungus ceases to act as a commensal and becomes pathogenic when the host presents susceptible factors. Due to the occurrence of antifungal resistance, in addition to their side effects and drug interactions, new therapies need to be used. And, the photodynamic therapy (PDT) is an intervention that has been an excellent option. Therefore, this article aims to address the use of PDT as monotherapy and in association with conventional therapy in the treatment of oral candidiasis, thus indicating whether this new therapeutic modality becomes a substitute or auxiliary technique in the treatment of oral candidiasis. Thusly, the literature review methodology based on books, scientific articles indexed in Scielo PubMed, Medline and Lilacs databases. Were used as inclusion criteria: books and articles published in the last 10 years, related to the topic of the article, made available in full. Exclusion Criteria: works not available in full and publications in annals of events. Eligibility Criteria: the reading of the title and abstract, where 2 books and 27 articles met the search criteria. The results showed that despite the use of PDT alone, it successfully fought oral candidiasis, the best results were obtained in the combination of therapies. It is concluded that currently the best treatment for oral candidiasis is the combination of PDT with traditional antifungals.

\section{INTRODUÇÃO}

A candidíase oral é a mais relevante infecção fúngica encontrada na cavidade oral humana, afetando principalmente a língua, o palato e a mucosa jugal. Sua etiologia está relacionada às espécies de leveduras do gênero Candida spp., um componente da microflora bucal normal. A Candida albicans é considerada a espécie mais patogênica, no entanto, outras espécies além da Candida albicans, também 
têm sido associadas a essa patologia, como: $C$. dubliniensis, C. parapsilosis, C. glabrata, C. krusei e C. tropicalis. Trata-se de uma infecção oportunista na qual a Candida spp. deixa de ter uma relação de comensalismo com o hospedeiro e torna-se patogênica na presença de fatores predisponentes, como por exemplo: hipossalivacão, uso de próteses dentárias, diabetes mellitus, uso prolongado de antibióticos e corticóides. Há uma relação direta entre a candidíase oral e pacientes com o sistema imunológico debilitado, já que a presença desta infecção demonstra falha imunológica nesses indivíduos. Algumas patologias sistêmicas predispõem o aparecimento da infecção fúngica, tais como AIDS (Síndrome da Imunodeficiência Adquirida), neoplasias malignas, síndromes metabólicas, entre outras (COSTA, 2011; NÚÑEZ; RIBEIRO; GARCEZ, 2013).

Existem três formas clinicas da candidíase oral: pseudomembranosa, eritematosa e hiperplásica. $\mathrm{Na}$ pseudomembranosa, observam-se placas brancas na mucosa bucal, facilmente removidas pela raspagem. Na forma clínica eritematosa, a lesão é vermelha, dolorida, ocorrendo no dorso da língua, observando-se regiões despapiladas e está relacionada ao uso prolongado de agentes antibacterianos de amplo espectro, que ao reduzir a quantidade de bactérias favorece a disseminação da Candida spp. E, a hiperplásica, em que são observadas principalmente na comissura labial e dorso da língua, placas brancas, espessas, não removidas pela raspagem (NÚÑEZ; RIBEIRO; GARCEZ, 2013).

A resistência dos microrganismos (bactérias, fungos e vírus) aos antimicrobianos é um tema preocupante e recorrente, sendo um sério problema à economia mundial e demandando a instituição de novas terapias, visto que para cada medicamento introduzido no mercado, em um curto espaço de tempo, já são reportados novos microrganismos resistentes (NÚÑEZ; RIBEIRO; GARCEZ, 2019).

Um dos fatores que pode explicar a resistência dos fungos às terapias atuais é o fato de existirem poucos medicamentos antifúngicos disponíveis para tratamento das infecções, o que aumenta a chance de desenvolvimento de resistência à terapia padrão. O mesmo problema reportado para os antifúngicos também é valido para os antibióticos, havendo elevada incidência e prevalência de resistência bacteriana aos antibióticos de diferentes classes, mesmo considerando a maior variedade quando comparado com os agentes antifúngicos. Outro fator é o fato do tratamento tópico resultar em melhora passageira, devido à diminuição da quantidade do antifúngico no local, ocasionada pela diluição salivar e pelas movimentações da língua (FREITAS, 2015).

Devido à ocorrência de resistência aos antifúngicos convencionais, influenciada pelo uso indiscriminado, além dos seus efeitos colaterais e interações medicamentosas, há a necessidade que novas modalidades terapêuticas sejam utilizadas. E, a Terapia Fotodinâmica (PDT, Photodynamic Therapy; ou aPDT, Antimicrobial Photodynamic Therapy) é uma intervenção que tem se destacado (NÚÑEZ; RIBEIRO; GARCEZ, 2019).

Eduardo (2015) afirma que a PDT é uma modalidade terapêutica que tem por intuito tratar infecções localizadas e superficiais, e, por esse motivo, não afeta os microrganismos nativos. Fundamenta-se na administração tópica de um fotossensibilizador (FS) sensível à luz seguida da irradiação com luz visível de comprimento de onda específico e a presença do oxigênio nas células, onde reações químicas acontecem e são gerados subprodutos que causam danos ao fungo. Então, ao se escolher o FS deve-se levar em conta a fonte de luz que será utilizada, visto que as características de um devem ser compatíveis com as do outro.

Em geral, o efeito fotodinâmico produz Espécies Reativas de Oxigênio (EROs), que atuam inicialmente sobre a membrana celular e, após o aumento da permeabilidade celular, penetram na célula fúngica e causam danos à outras organelas intracelulares. Portanto, esta terapia mostra-se uma excelente opção de tratamento da candidíase oral (FREITAS, 2015).

Portanto, este artigo tem como objetivo abordar o uso da PDT como monoterapia e em associação com a terapia convencional no tratamento da candidiase oral, indicando desta forma se esta nova modalidade terapêutica vem a ser uma técnica substituta ou auxiliar no tratamento da candidíase oral.

\section{MÉTODOS}

Este trabalho foi desenvolvido por meio de uma revisão de literatura baseada em livros, artigos científicos indexados nas bases da Scientific Electronic Library Online (Scielo), US Nacional Library of Medicine National Institutes of Health (PubMed), Medical Literature Analysis and Retrieval System Online (Medline) e Literatura Latino-americana e do Caribe em Ciências da Saúde (Lilacs). Esta revisão apresentou como critérios de inclusão: livros e artigos publicados nos últimos 10 anos (2019-2009), tanto na língua portuguesa como inglesa, relacionados à temática do artigo. Critérios de exclusão: trabalhos não disponíveis na íntegra e publicações em anais de eventos. Critérios de qualificação: a leitura do título e do resumo.

\section{TERAPIA CONVENCIONAL X PDT}

Comparando-se essas duas terapias destacam-se as seguintes desvantagens da terapia convencional: possibilidade de resistência do fungo, interações 
medicamentosas e os seguintes efeitos colaterais na terapia tópica: possibilidade de ocorrência de náusea e vômito. Já a terapia sistêmica apresenta como desvantagens: possibilidade de danos aos rins, fígado, problemas gastrointestinais e depressão da medula óssea (FREITAS, 2015).

Já a PDT não apresenta efeitos colaterais ao paciente, tem custo acessível, apresenta ausência de interações medicamentosa e impossibilita o desenvolvimento de resistência microbiana, já que atua sobre diversos alvos intracelulares, diferentemente da terapia sintética que atua em um alvo especifico do fungo (FREITAS, 2015).

\section{TERAPIA FOTODINÂMICA (PHOTODYNAMIC THERAPY - PDT)}

A terapia fotodinâmica usada no tratamento da candidíase e de outras patologias orais e periorais baseia-se na administração tópica de um fotossensibilizador (FS) sensível à luz, seguida da irradiação com luz visível (Figura 1).

Figura 1. Aplicação do FS e irradiação com laser de baixa potência em paciente com herpes simples.

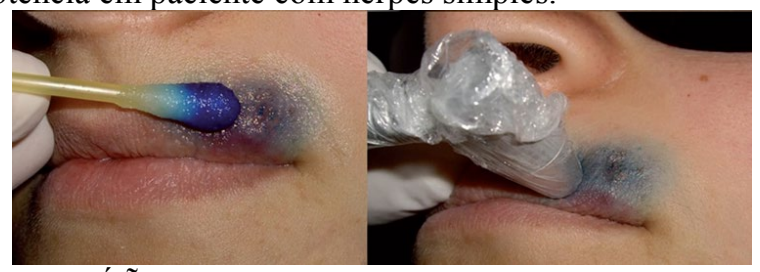

Fonte: NÚÑEZ; RIBEIRO; GARCEZ (2019)

Cada FS tem absorção máxima e somente é ativado por determinado comprimento de onda de luz que corresponde a uma cor específica no espectro visível de luz, conforme o Quadro 1 e Figura 2 (NÚÑEZ; RIBEIRO; GARCEZ, 2013).

Quadro 1. Exemplos de alguns Fotossensibilizadores e suas bandas de absorção.

\begin{tabular}{|l|l|}
\hline $\begin{array}{c}\text { Fotossensibilizador } \\
\text { (FS) }\end{array}$ & \multicolumn{1}{|c|}{$\begin{array}{c}\text { Comprimento de onda } \\
\text { absorvida pelo FS } \\
\text { (nanômetros [nm]) }\end{array}$} \\
\hline Azul de metileno & $610-660$ (vermelho) \\
\hline Azul de toluidina & $\begin{array}{l}590-630 \\
\text { (alaranjado/vermelho) }\end{array}$ \\
\hline Rosa bengala & $\begin{array}{l}480-600 \\
\text { (azul ao vermelho) }\end{array}$ \\
\hline Cristal violeta & $\begin{array}{l}420-650 \\
\text { (azul ao vermelho) }\end{array}$ \\
\hline
\end{tabular}

Fonte: NÚÑEZ; RIBEIRO; GARCEZ (2013)
Figura 2. LED's com emissão de diversos comprimentos de onda.

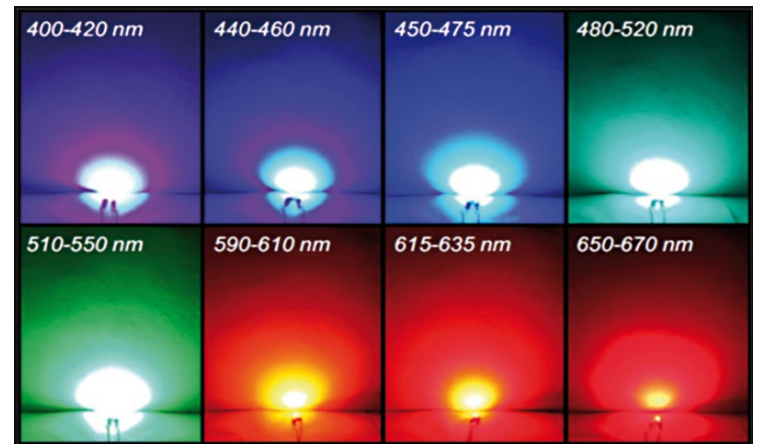

Fonte: NÚÑEZ; RIBEIRO; GARCEZ (2019)

\section{Fotossensibilizadores (FS)}

Os fotossensibilizadores tratam-se de corantes biológicos. Sua utilização é considerada segura, pois, são utilizados há mais de cem anos na medicina. Foram realizados diversos estudos com essas substâncias, demonstrando sua baixa toxicidade. Na PDT, a chance de toxicidade é diminuída ainda mais, devido os corantes serem utilizados topicamente, diminuindo o tempo de exposição, facilitando o controle da quantidade pelo profissional, além dos principais corantes utilizados na PDT serem hidrofílicos, sendo rapidamente eliminados do organismo humano (NÚÑEZ; RIBEIRO; GARCEZ, 2019).

Dentre os diversos FS's empregados na PDT destacam-se: o azul de metileno, azul de toluidina, porfirinas, clorinas, ftalocianinas, dentre outros. E, dentre estes, o Azul de Metileno (AM) é o mais utilizado e conhecido. Devido a sua máxima absorção de luz se situar no comprimento de onda de 610 a $660 \mathrm{~nm}$, a fonte de luz a ser utilizada com esse FS deve emitir fótons vermelhos para que ocorra sua fotoativação (NÚÑEZ; RIBEIRO; GARCEZ, 2013; EDUARDO, 2015).

\section{Fontes de luz}

Existem várias fontes de luz que podem ser utilizadas na PDT, mas, atualmente as principais são: o diodo emissor de luz (LED) e os lasers de baixa potência (Figuras 3 e 4). E, há uma tendência de preferência pelos lasers no tratamento da candidíase, devido possuírem as seguintes características: emissão de fótons em um único comprimento de onda (monocromaticidade), na mesma direção (colimação) e em sincronia (coerência). Enquanto, os LEDs possuem apenas monocromaticidade (EDUARDO, 2015).

Os lasers de baixa potência também conhecidos como laser terapêutico, não ablativo e de baixa intensidade, promovem reparação tecidual, diminuição da inflamação e analgesia. Diferentemente dos lasers de alta potência, os de 
baixa intensidade não aumentam a temperatura no tecido, não possuindo efeito antimicrobiano quando usado de maneira isolada. Entretanto, estes lasers, quando utilizados em associação com agentes fotossensibilizadores proporcionam uma grande capacidade de redução de microrganismos na aplicação da terapia fotodinâmica (EDUARDO et al., 2015).

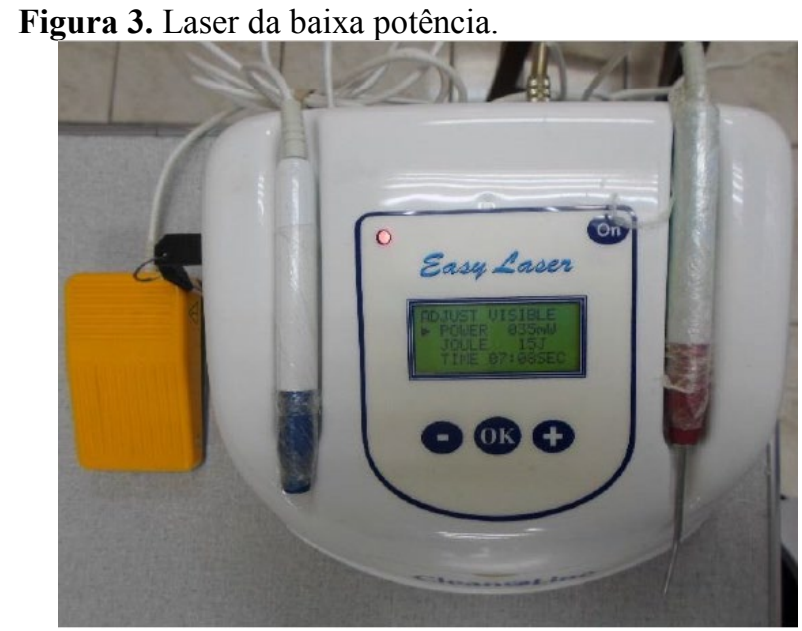

Fonte: FREITAS (2015)

Figura 4. LED (Diodo Emissor de Luz).

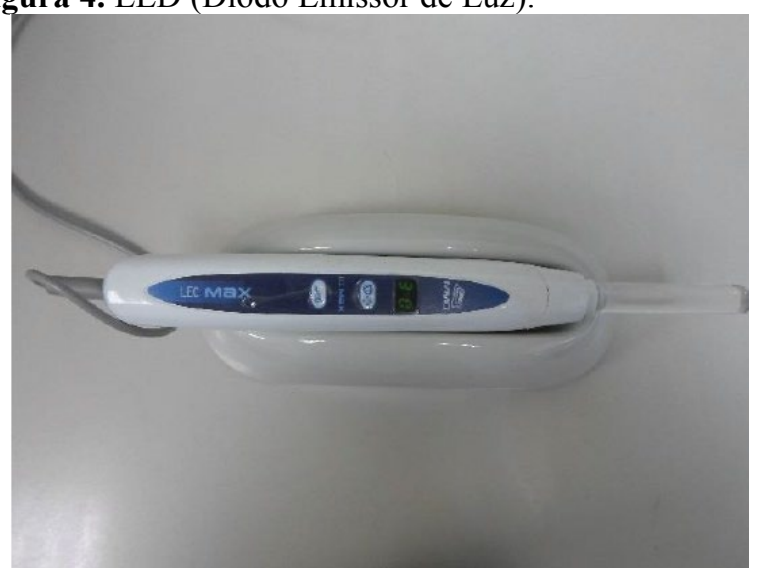

Fonte: FREITAS (2015)

\section{MECANISMO DE AÇÃO DA PDT}

O efeito fotodinâmico é obtido pela reação de um FS, uma fonte de luz e o oxigênio presente nas células, podendo levar a eliminação da célula fúngica através de três principais mecanismos: Reação do tipo I, onde o FS transfere elétrons ou hidrogênio às células fúngicas, produzindo radicais livres; Reação do tipo II, onde é produzido oxigênio singleto, através da transferência de energia ao oxigênio pelo FS; e, por fim, através da indução da morte programada do fungo (apoptose), em que o efeito fotodinâmico causa danos a membrana plasmática, ativando proteínas executoras da apoptose e bloqueando os mecanismo de defesa do fungo a esse processo. Dentre esses mecanismos, o mais comum e o principal causador da morte do fungo é a Reação do tipo II, no qual as espécies reativas de oxigênio produzidas danificam a parede celular, as organelas intracelulares e o DNA fúngico (QUEIROGA, 2010).

\section{O USO DA TERAPIA FOTODINÂMICA DE MANEIRA ISOLADA E EM ASSOCIAÇÃO COM A TERAPIA CONVENCIONAL NO TRATAMENTO DA CANDIDIASE ORAL}

\section{Monoterapia}

Carmello et al. (2019) avaliaram a eficácia da PDT mediada por ftalocianina de cloro-alumínio encapsulada em nanoemulsões catiônicas (CIAIP-NE) para tratar a candidíase oral in vivo e seu efeito na adesão e formação de biofilme de Candida albicans. Para isso, camundongos foram imunossuprimidos e inoculados com $C$. albicans para produzir candidíase oral. PDT e Nistatina foram aplicados por 5 sessões sucessivas como monoterapia em dois grupos. Ambos os tratamentos reduziram a capacidade de adesão e formação de biofilme de $C$. albicans. A PDT mediada por CIAIP-NE foi eficaz na redução de $C$. albicans e também no tratamento da candidíase oral induzida em camundongos.

Silva et al. (2016) desenvolveram um estudo que teve como intuito identificar as espécies de leveduras isoladas de crianças não expostas, expostas ao HIV e portadoras de AIDS, e verificar a eficácia da terapia fotodinâmica com laser de baixa potência sobre as espécies de leveduras pertencentes ao gênero Candida. As cepas foram submetidas à Terapia Fotodinâmica com laser de baixa potência de $660 \mathrm{~nm}$ e corante AM em diferentes tempos de irradiação (90, 180 e 282 segundos). Os resultados mostraram que a espécie isolada mais prevalente nos grupos estudos foi Candida albicans. Houve diminuição significante de UFC/ml de Candida spp. $(p<0,05)$ em todos os grupos tratados com terapia fotodinâmica, em comparação com os controles. A PDT foi eficaz na inativação de Candida spp. isoladas a partir da cavidade oral de crianças não expostas, expostas ao HIV e portadoras da AIDS, com o melhor tempo de irradiação sendo o de $282 \mathrm{seg}$.

Carmello et al. (2016) avaliaram a fotoinativação de Candida albicans em um modelo murino de candidíase oral usando ftalocianina de cloro-alumínio (CIAIP) encapsulada em nanoemulsões catiônicas (NE) e ftalocianina de cloroalumínio (CIAIP) diluída em DMSO (dimetilsulfóxido ou sulfóxido de dimetilo) como fotossensibilizador. Setenta e cinco ratos swiss fêmeas de 6 semanas de idade foram imunossuprimidos e inoculados com $C$. albicans para induzir candidíase oral. A PDT foi realizada na língua pela aplicação 
dos fotossensibilizadores e luz LED (100 J cm2 - $660 \mathrm{~nm})$. Vinte e quatro horas e 7 dias após os tratamentos, a avaliação microbiológica foi realizada através da recuperação de $C$. albicans da língua dos animais (UFC $\mathrm{ml}(-1)$ ). Em seguida, os ratos foram sacrificados e as línguas foram removidas cirurgicamente para análise histológica e biomolecular de citocinas anti-inflamatórias. Os dados foram analisados por ANOVA, seguido do teste post hoc de Tukey. A PDT mediada por CIAIP-NE reduziu a quantidade de $C$. albicans recuperados da língua quando comparados ao grupo controle $(P<0,05)$. $A$ PDT não promoveu efeitos adversos no tecido da língua. Sete dias após o tratamento, todos os animais estavam completamente saudáveis. Em resumo, a PDT mediada por ftalocianina de cloro-alumínio aprisionada em nanoemulsões catiônicas foi eficaz na redução de $C$. albicans recuperada das lesões orais de camundongos imunocomprometidos (figura 5).

Figura 5. Aspecto lingual após inoculação com $C$. albicans e depois de 7 dias de tratamento com PDT mediada por ClAlP-NE.

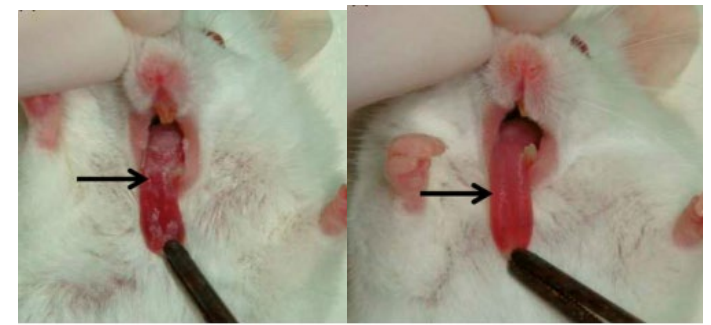

Fonte: CARMELLO et al. (2016)

Sousa et al. (2015) avaliaram o efeito da terapia fotodinâmica na inativação de células planctônicas de Candida albicans, empregando AM a $150 \mu \mathrm{g} / \mathrm{mL}$ como fotossensibilizador. Alíquotas de $100 \mu \mathrm{g} / \mathrm{mL}$ da suspensão do fungo na concentração de $1 \times 10^{6}$ células $/ \mathrm{mL}$ foram semeadas em placas de microtitulação com 96 poços, onde foi depositado o mesmo volume de AM, permanecendo por um período de pré-irradiação de $5 \mathrm{~min}$. Em seguida, aplicou-se luz laser de baixa potência (comprimento de onda: $660 \mathrm{~nm}$, potência: $100 \mathrm{~mW}$ e dose: $426 \mathrm{~J} / \mathrm{cm} 2$ ) por $128 \mathrm{~s}$, utilizando um Laser semicondutor portátil. Na concentração de $150 \mu \mathrm{g} / \mathrm{mL}$ e tempo de 5 min de incubação, a redução foi significativa $(p<0,05)$. Na ausência de irradiação, o AM não produziu redução de UFC/mL. A Terapia Fotodinâmica apresentou efeito antifúngico contra $C$. albicans, podendo ser utilizada como coadjuvante ao tratamento convencional.

Pereira et al. (2015) avaliaram o potencial de inativação fotodinâmica em cultura planctônica e biofilmes de cepas de Candida. Um total de 48 isolados clínicos de Candida de indivíduos usando próteses maxilares removíveis com estomatite protética (EP) foram incluídos no estudo. Os efeitos da eritrosina (ER, $200 \mu \mathrm{M})$ e um LED verde $(\lambda 532 \pm 10$ $\mathrm{nm}, 237 \mathrm{~mW} / \mathrm{cm} 2$ e 42,63 J/cm2) foram avaliados. As cepas de Candida foram avaliados antes e após o processo da PDT em células derivadas de biofilme e ensaios planctônicos. Todas as espécies de Candida foram suscetíveis a ER e LED verde. Estes resultados sugerem que a adição de PDT é eficaz na redução de certas espécies de Candida, além de diminuir sua patogenicidade.

Dovigo et al. (2013) estudaram a fotoinativação de Candida albicans em um modelo murino de candidíase oral, usando a curcumina como fotossensibilizador. Quarenta camundongos imunossuprimidos foram inoculados por via oral com C. albicans e, após cinco dias, receberam curcumina tópica (20, 40 e $80 \mu \mathrm{M})$ e iluminação com luz LED. Todas as exposições à curcumina com luz LED causaram uma redução significativa na viabilidade de $C$. albicans após a PDT. Concluiuse que a PDT mediada por curcumina mostrou-se eficaz na inativação in vivo de $C$. albicans sem danificar o tecido hospedeiro de camundongos.

Alves (2013) avaliou os efeitos da PDT, mediada pelo Photodithazine ${ }^{\circledR}$ (PDZ) e luz LED, sobre $C$. albicans resistente a fluconazol em um modelo de candidose oral induzida. A dose de luz selecionada foi de $37,5 \mathrm{~J} / \mathrm{cm} 2$ e o tempo de iluminação foi de 14 minutos. Os animais foram sacrificados e as línguas foram removidas cirurgicamente para análise histológica. Duas colônias de cada animal foram isoladas da placa de cultura para avaliação dos fatores de virulência: adesão e formação de biofilme em superfície abiótica, formas filamentares de crescimento e produção de exoenzimas. Os dados foram analizados por ANOVA $(P<0,05)$. Os resultados demonstraram que a PDT resultou em redução significativa de C. albicans resistente a fluconazol em relação ao grupo controle positivo. A PDT foi efetiva na inativação da C. albicans resistente a fluconazol, sem causar efeitos adversos no tecido lingual.

Scwingel et al. (2012) avaliaram a eficácia do PDT no tratamento da candidíase em pacientes infectados pelo HIV. Vinte e um pacientes foram divididos em três grupos. O grupo controle (GC) foi tratado com a medicação convencional para candidíase (fluconazol $100 \mathrm{mg} /$ dia durante 14 dias). 0 grupo laser (LG) foi submetido à laserterapia de baixo nível (LLLT), comprimento de onda $660 \mathrm{~nm}$, potência de $30 \mathrm{~mW}$ e fluência de $7,5 \mathrm{~J} / \mathrm{cm} 2$, em contato com a mucosa durante 10 segundos no ponto afetado. $O$ grupo PDT foi tratado com a combinação de laser de baixa potência e azul de metileno a $450 \mu \mathrm{g} / \mathrm{mL}$. O tempo de pré-irradiação foi de 1 minuto. Os resultados mostraram que o fluconazol foi eficaz; no entanto, não impediu o retorno da candidíase a curto prazo. O LLLT por si só não mostrou redução em Candida spp. O PDT erradicou $100 \%$ das colônias desse fungo e os pacientes não apresentaram recorrência de candidíase até 30 dias após a 
irradiação. Esses achados sugerem que o PDT é uma abordagem potencial para o tratamento da candidíase oral em pacientes infectados pelo HIV.

Mima et al. (2012) em um ensaio clínico randomizado, a eficácia clínica e micológica da terapia fotodinâmica (PDT) foi comparada com a da terapia antifúngica tópica para o tratamento de EP. Os pacientes foram divididos aleatoriamente em um dos dois grupos ( $n=20$ cada); no grupo nistatina (NYT), os pacientes receberam tratamento tópico com nistatina (100.000 UI) quatro vezes ao dia por 15 dias; no grupo PDT, a prótese e o palato dos pacientes foram pulverizados com $500 \mathrm{mg} / \mathrm{L}$ de Photogem e após $30 \mathrm{~min}$. da incubação, foram iluminados por luz de diodo emissor de luz a $455 \mathrm{~nm}$ (37,5 e $122 \mathrm{~J} / \mathrm{cm} 2$, respectivamente) três vezes por semana durante 15 dias. Os grupos NYT e PDT apresentaram taxas de sucesso clínico de $53 \%$ e $45 \%$, respectivamente. A PDT foi tão eficaz quanto à nistatina tópica no tratamento da EP (figura 6).

Figura 6. Palato de paciente com EP antes e após o tratamento com PDT.

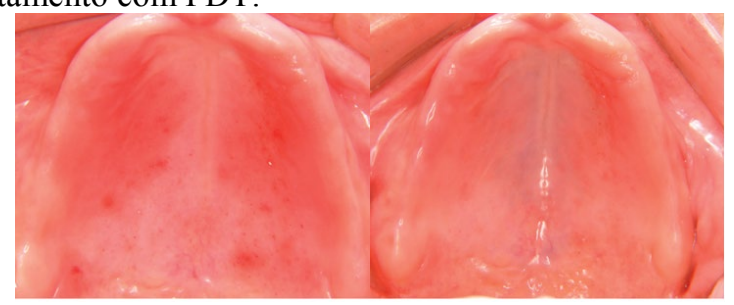

Fonte: MIMA et al. (2012)

Mima et al. (2011) descreveram em um relatório cinco casos de EP tratados com PDT. Os indivíduos foram submetidos a 6 sessões de PDT 3 vezes por semana durante 15 dias. Em cada sessão, as próteses e palatos dos pacientes foram pulverizados com $500 \mathrm{mg} / \mathrm{L}$ de Photogem e, após 30 minutos de incubação, irradiados por fonte de luz de diodo emissor de luz a $455 \mathrm{~nm}$ (37,5 e $122 \mathrm{~J} / \mathrm{cm} 2$, respectivamente). Quatro pacientes apresentaram resolução clínica da EP (sem inflamação) após as sessões de PDT, e um indivíduo demonstrou redução na inflamação palatal. A PDT parece ser um tratamento alternativo para o EP.

Martins et al. (2011) avaliaram os efeitos da terapia fotodinâmica na patogenicidade de $C$. albicans. Cinquenta e seis ratos foram submetidos ao desenvolvimento de candidíase no dorso lingual por inoculação de $C$. albicans. Após 5 dias, diferentes tratamentos foram administrados: laser e azul de metileno $(\mathrm{L}+\mathrm{P}+)$; somente laser $(\mathrm{L}+\mathrm{P}-)$; fotossensibilizador apenas ( $(-P+)$; e solução fisiológica apenas (L-P-). Amostras da cavidade bucal foram coletadas para contagem de unidades formadoras de colônias por $\mathrm{mL}$ e valores de atividades enzimáticas foram analisadas por análise de variância (ANOVA) e teste de Tukey. A PDT reduziu as lesões microscópicas da candidíase experimental em ratos e inibiu a atividade proteinase de $C$. albicans.

Queiroga et al. (2011) avaliaram a eficácia da PDT na fotoinativação de espécies de Candida utilizando AM e irradiação com laser de diodo (660 nm, $40 \mathrm{~mW}$ ). Foram obtidas suspensões de espécies de Candida contendo 106 $\mathrm{UFC} / \mathrm{ml}$, transferidas para placas de 96 furos e expostas a 03 doses de luz laser (60 J/cm2, $120 \mathrm{~J} / \mathrm{cm} 2,180 \mathrm{~J} / \mathrm{cm} 2)$ na presença de AM. As três doses avaliadas determinaram inativação significativa de Candida spp. A dose de $180 \mathrm{~J} / \mathrm{cm} 2$ foi a mais eficaz, inativando 78\% de UFC/ml. Na dose de 180 $\mathrm{J} / \mathrm{cm} 2$, C. albicans foi a espécie mais suscetível. A PDT demonstrou eficácia na inativação de Candida spp.

Gendreau e Loewy (2011) realizaram um relato de caso, em que cinco pacientes com EP foram submetidos a seis sessões de PDT durante 2 semanas (três sessões por semana). Em cada sessão, Photogem (500 mg/L) foi aplicado tanto no palato como nas próteses do paciente, que, após $30 \mathrm{~min}$, foram iluminados por LED ( $\approx 455 \mathrm{~nm}$ ) a 122 e 37,5J/cm2, respectivamente. Em quatro pacientes foi observada resolução clínica das lesões de estomatite protética, e um dos pacientes apresentou diminuição das lesões. Todos pacientes apresentaram redução de crescimento de Candida spp. tanto do palato como da prótese imediatamente após o tratamento, comparado com crescimento observado antes do tratamento (figura 7).

Figura 7. Uso da PDT no palato e na prótese de paciente com EP.

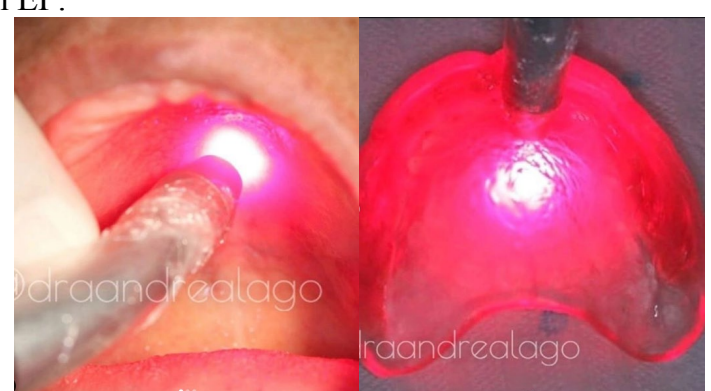

Fonte: LAGO (2019)

Mima et al. (2010) avaliaram a eficácia da PDT na inativação de Candida albicans in vivo. Setenta e um camundongos swiss, fêmeas, com 6 semanas de idade, foram imunossuprimidos, solubilizaram tetraciclina à água potável e, em seguida, esfregaram oralmente com uma suspensão de $C$. albicans (107 UFC/mL). Quatro dias após a inoculação oral, a PDT foi realizada no dorso da língua após administração tópica de Photogem a 400, 500 ou 1000 mg/L e seguida 30 minutos depois por iluminação com luz LED (305 J/cm²) em 455 ou $630 \mathrm{~nm}$ ( $\mathrm{n}=5$ cada). A PDT resultou em uma redução 
significativa em $C$. albicans recuperada da língua $(P<0,001)$ quando comparada com camundongos do grupo controle positivo. Não houve diferença entre as concentrações de Photogem e comprimentos de onda da luz LED utilizados. A avaliação histológica da língua revelou que a PDT não causa efeitos adversos significativos na mucosa local.

\section{Politerapia}

Afroozi et al. (2019) investigaram a eficácia da PDT mediada por indocianina verde (ICG) em combinação com nistatina suspensão oral (PDT+nistatina) para o tratamento de estomatite dentária em comparação com a terapia antifúngica de rotina apenas com nistatina. Neste ensaio clínico randomizado duplo-cego, 66 pacientes foram divididos aleatoriamente nos grupos PDT+nistatina $(n=33)$ e nistatina ( $n=33$ ), ambos os grupos foram tratados 3 vezes ao dia (15 dias) com nistatina suspensão e a PDT foi realizada duas vezes, uma vez por semana no grupo PDT + nistatina. Resumidamente, o ICG foi aplicado na lesão palatal e a irradiação com laser foi realizada com laser de diodo $(810 \mathrm{~nm}$, $56 \mathrm{~J} / \mathrm{cm} 2$ ). O tratamento do paciente com nistatina ou PDT+nistatina reduziu a extensão das lesões. A redução média alcançada no grupo PDT+nistatina foi significativamente maior que a nistatina sozinha. A PDT mediada por ICG em combinação com a suspensão de nistatina pode melhorar a característica clínica da estomatite dentária sem efeitos adversos; portanto, poderia ser usado como uma alternativa à terapia antifúngica atualmente disponível em que se usa apenas a nistatina.

Hsieh et al. (2018) testaram a PDT utilizando curcumina e luz azul como uma terapia independente e em combinação com o tratamento com fluconazol. Os ensaios de viabilidade e análise morfológica foram usados para avaliar a eficácia do tratamento com $C$. albicans. Os resultados mostraram que o tratamento com fluconazol diminuiu a viabilidade de $C$. albicans planctônicos, mas a diminuição não foi tão pronunciada em $C$. albicans porque sua forma de biofilme foi marcadamente mais resistente ao antifúngico. A PDT foi efetiva contra biofilmes de $C$. albicans de maneira isolada, e quando combinado com fluconazol, a PDT inibiu significativamente $C$. albicans em maior medida. Este estudo sugere que a adição de PDT ao fluconazol para tratar a infecção por $C$. albicans aumenta sua eficácia e pode potencialmente ser usada clinicamente.

Huang et al. (2018) realizaram um estudo em que foi explorado a possibilidade de combinar a PDT e agentes antifúngicos para aumentar a eficácia terapêutica contra Candida albicans e isolados clínicos resistentes a fármacos. Foi descoberto que as células planctônicas que sobreviveram à PDT mediada pelo azul de toluidina $O$ (TBO) foram significativamente suscetíveis ao fluconazol nas primeiras duas horas após a PDT. Ainda foi descoberto que uma combinação de PDT com caspofungina resultou na morte completa de biofilmes em comparação com aqueles tratados com caspofungina ou PDT sozinho. Estes resultados indicam claramente que a combinação de PDT e agentes antifúngicos pode ser um promissor tratamento contra infecções por $C$. albicans.

Hidalgo (2018) realizou um estudo que avaliou a eficácia da PDT associada à Nistatina no tratamento de candidose oral induzida em camundongos infectados com $C$. albicans resistente a fluconazol. A análise macroscópica revelou remissão das lesões orais, e a análise histológica demonstrou expressiva redução da reação inflamatória no tecido que apresentou características histológicas de normalidade. Dessa forma, pode-se concluir que a combinação de terapias é uma alternativa para o tratamento de candidose induzida com cepas de $C$. albicans resistente ao fluconazol.

Freire et al. (2016) investigaram os efeitos da PDT em $C$. albicans como biofilmes cultivados in vitro e também em um modelo de camundongo imunossuprimido de infecção por candidíase oral. Os corantes fenotiazínicos, azul de metileno (AM) e novo azul de metileno (NAM) foram usados como FS's, combinados ou não com iodeto de potássio (IP) e laser vermelho $(660 \mathrm{~nm})$ em quatro doses diferentes de luz (10 J, $20 \mathrm{~J}, 40 \mathrm{~J}$ e $60 \mathrm{~J}$ ). Após 5 dias de tratamento, a doença foi praticamente erradicada, especialmente utilizando AM mais IP com $40 \mathrm{~J}$. Este estudo sugere que o IP pode potencializar a PDT da infecção fúngica usando AM e pode ser uma nova abordagem promissora para o tratamento de candidíase.

Lyon et al. (2016) avaliaram o efeito combinado de PDT e fluconazol contra $C$. albicans resistentes e Candida glabrata. As leveduras foram submetidas ao azul de metileno-PDT (AMPDT) em concentrações sub-inibitórias. No presente trabalho, o AM-PDT combinado ao fluconazol foi mais eficiente na inibição de $C$. albicans e $C$. glabrata do que em cada tratamento isolado, sendo possível inferir que os tratamentos são sinérgicos.

Snell et al. (2012) procuraram identificar compostos em uso clínico que aumentassem o estresse oxidativo causado pela PDT, contribuindo para a própria formação de espécies reativas de oxigênio (ERO's). Com base na capacidade do antifúngico miconazol de induzir ERO's em Candida, testaram vários antifúngicos azólicos quanto à sua capacidade de aumentar a PDT in vitro. Embora o miconazol e o cetoconazol estimulem a produção de ERO's em $C$. albicans, apenas o miconazol aumentou a morte de $C$. albicans e induziu ação fungistática prolongada em organismos que sobreviveram à PDT usando a porfirina TMP-1363 e o AM como FS's. Os dados sugerem que o miconazol pode ser usado para aumentar a eficácia da PDT contra C. albicans, e seu mecanismo de ação 
provavelmente é multifatorial.

\section{CONSIDERAÇÕES FINAIS}

Atualmente, de acordo com os vários estudos realizados o tratamento eficaz para a candidíase oral é a combinação da PDT com a os antifúngicos tradicionais. O que mostra que esta opção terapêutica da Candida não deve ser considerada uma alternativa substituta as terapias convencionais, mas, um complemento à terapia sintética. Corroborando com os estudos avaliados, onde a politerapia se mostrou mais eficaz que a monoterapia. Com o uso da PDT ocorre uma redução da população de fungos no local da infecção, consequentemente será utilizada uma menor quantidade de antifúngico, diminuindo consideravelmente a chance de desenvolvimento de resistência dos microrganismos à farmacologia atual além de aumentar a eficácia no combate a esse tipo de infecção.

\section{REFERÊNCIAS}

AFROOZI, B; ZOMORODIAN, K; LAVAEE, F; SHAHRABADI, Z; MARDANI, M. Comparison of the efficacy of indocyanine green-mediated photodynamic therapy and nystatin therapy in treatment of denture stomatitis. Photodiagnosis and Photodynamic Therapy, v. 27, p. 193197, 2019. Disponível em:< https://www.ncbi.nlm.nih.gov/pubmed/31185323>.

Acesso em: 25 ago. 2019. doi: 10.1016/j.pdpdt.2019.06.005.

ALVES, F. Estudo in vivo dos efeitos da terapia fotodinâmica, mediada pelo Photothazine ${ }^{\circledR}$ e luz led, sobre Cândida Albicans resistente a Fluconazol. 2013. Dissertação (mestrado) - Universidade Estadual Paulista Julio de Mesquita Filho, Faculdade de Odontologia de Araraquara, Araraquara, 2013. Disponível em: <http://hdl.handle.net/11449/97271>. Acesso em: 07 set. 2019.

CARMELLO, J. C; ALVES, F; BASSO, F. G; COSTA, C. A. S; TEDESCO, A. C; PRIMO, F. L; MIMA, E. G. O; PAVARINA, A. C. Antimicrobial photodynamic therapy reduces adhesion capacity and biofilm formation of Candida albicans from induced oral candidiasis in mice. Photodiagnosis and Photodynamic Therapy, v. 27, p. 402-407, 2019. Disponível em:

https://www.ncbi.nlm.nih.gov/pubmed/31284075>.

Acesso em: 28 ago. 2019. https://doi.org/10.1016/j.pdpdt.2019.06.010.

CARMELLO, J. C; ALVES, F; RIBEIRO, A; BASSO, F. G; COSTA, C. A. S; TEDESCO, A. C; PRIMO, F. L; MIMA, E. G. O; PAVARINA, A. C. In vivo photodynamic inactivation of Candida albicans using chloro-aluminum phthalocyanine. Oral Diseases, v. 22, n. 5, p. 415-422, 2016. Disponível em:< https://www.ncbi.nlm.nih.gov/pubmed/26914185>.

Acesso em: 28 ago. 2019. doi: 10.1111/odi.12466.

COSTA, A. C. B. P. Avaliação da Terapia Fotodinâmica em Candida albicans in vitro e in vivo. 2011. Dissertação (Mestrado em Biopatologia Bucal) - Faculdade de Odontologia de São José dos Campos, Universidade Estadual Paulista "Júlio de Mesquita Filho", São José dos Campos, 2011. Disponível em: <https://repositorio.unesp.br/handle/11449/95047>.

Acesso em: 11 mar. 2019.

DOVIGO, L. N; CARMELLO, J. C; COSTA, C. A. S; VERGANI, C. E; BRUNETTI, I. L; BAGNATO, V. S; PAVARINA, A. C. Curcuminmediated photodynamic inactivation of Candida albicans in a murine model of oral candidiasis. Medical Mycology, v. 51, p. 243-251, 2013. Disponível em:< https://www.ncbi.nlm.nih.gov/pubmed/22934533>.

Acesso em: 28 ago. 2019. https://doi.org/10.3109/13693786.2012.714081.

EDUARDO, C. P; BELLO-SILVA, M. S; RAMALHO, K. M; LEE, E. M. R; ARANHA, A. C. C. A terapia fotodinâmica como benefício complementar na clínica odontológica. Revista Associação Paulista Cirurgiões Dentistas, v. 69, n. 3, p. 226235, 2015. Disponível em: $<$ http://revodonto.bvsalud.org/scielo.php?script=sci_artt ext\&pid=S000452762015000200004\&lng=pt\&nrm=iso $>$. Acesso em: 22 mar. 2019.

FREIRE, F; FERRARESI, C; JORGE, A. O. C; HAMBLIN, M. R. Photodynamic therapy of oral Candida infection in a mouse model. Journal of Photochemistry and Photobiology B: Biology, v. 159, p. 161-168, 2016. Disponível em < https://www.sciencedirect.com/science/article/pii/S1011 134416301464>. Acesso em: 25 ago. 2019. https://doi.org/10.1016/j.jphotobiol.2016.03.049.

FREITAS, L. S. F. Efeito antimicrobiano de múltiplas sessões de terapia fotodinâmica sobre biofilmes de Candida spp. Formados in vitro. 2015. Tese (Doutorado em Biopatologia Bucal) - Instituto de Ciência e Tecnologia, Universidade Estadual Paulista, São José dos Campos, 2015. Disponível em:

<https://repositorio.unesp.br/handle/11449/127698>. Acesso em: 09 mar. 2019.

GENDREAU, L; LOEWY, Z. G. Epidemiology and etiology of denture stomatitis. Journal Prosthodontics, v. 20, p. 251260, 2011. Disponível em: < https://www.ncbi.nlm.nih.gov/pubmed/21463383>. Acesso em: 28 dez. 2019.

HIDALGO, K. J. R. Eficácia da terapia fotodinâmica antimicrobiana associada a nistatina no tratamento de 
candidose oral em camundongos infectados com Candida albicans resistente a fluconazol. 2018. Dissertação (Mestrado em Reabilitação Oral) - Faculdade de Odontologia da UNESP, Araraquara, 2018. Disponível em: <https://repositorio.unesp.br/handle/11449/180213>. Acesso em: 09 mar. 2019.

HSIEH, Y. H; ZHANG, J. H; CHUANG, W. C; YU, K. H; HUANG, X. B; LEE, Y. C; LEE, C. I. An in Vitro Study on the Effect of Combined Treatment with Photodynamic and Chemical Therapies on Candida albicans. International journal of molecular sciences, v. 19, n. 2, p. 337, 2018. Disponível em:

<https://www.ncbi.nlm.nih.gov/pubmed/29364155>.

Acesso em: 15 mar. 2019

HUANG, M. C; SHEN, M; HUANG, Y. J; LIN, H. C; CHEN, C. T. Photodynamic Inactivation Potentiates the Susceptibility of Antifungal Agents against the Planktonic and Biofilm Cells of Candida albicans. International journal of molecular sciences, v. 19, n. 2, p. 434, 2018. Disponivel em:

<https://www.ncbi.nlm.nih.gov/pubmed/29389883>.

Acesso em: 25 mar. 2019.

LAGO, A. D. N. Tratamento de estomatite protética com terapia fotodinâmica. São Luís, 09 abr. 2019. Instagram: @draandrealago. Disponível em: $<$ https://www.instagram.com/p/BwDgdDiJlgn/>. Acesso em: 02 dez. 2019.

LYON, J. P; CARVALHO, C. R; REZENDE, R. R; LIMA, C. J; SANTOS, F. V; MOREIRA, L. M. Synergism between fluconazole and methylene blue-photodynamic therapy against fluconazole-resistant Candida strains. Indian Journal of Medical Microbiology, v. 34, n. 4, p. 506-508, $2016 . \quad$ Disponível em: <http://www.ijmm.org/text.asp?2016/34/4/506/195351 >. Acesso em: 05 dez. 2019

MARTINS, J. S; JUNQUEIRA, J. C; FARIA, R. L; SANTIAGO, N. F; ROSSONI, R. D; COLOMBO, C. E; JORGE, A. O. Antimicrobial photodynamic therapy in rat experimental candidiasis: evaluation of pathogenicity factors of Candida albicans. Oral Surgery, Oral Medicine, Oral Pathology, Oral Radiology and Endodontology, v. 111, n. 1, p. 71-77. Disponivel em:< https://www.ncbi.nlm.nih.gov/pubmed/21176823>.

Acesso em: 25 ago. 2019. doi: 10.1016/j.tripleo.2010.08.012.

MIMA, E. G. O; VERGANI, C. E; MACHADO, A. L; MASSUCATO, E. M. S; COLOMBO, A. L; BAGNATO, V. S; PAVARINA, A. C. Comparison of Photodynamic Therapy versus conventional antifungal therapy for the treatment of denture stomatitis: a randomized clinical trial. Clinical microbiology and infection, v. 18, p. E380-E388, 2012.
Disponível

em:

$<$ https://www.sciencedirect.com/science/article/pii/S1198 743X14610776>. Acesso em: 25 ago. 2019. https://doi.org/10.1111/j.1469-0691.2012.03933.x.

MIMA, E. G. O; PAVARINA, A. C; DOVIGO, L. N; VERGANI, C. E; COSTA, C. A; KURASHI, C; BAGNATO, V. S. Susceptibility of Candida albicans to photodynamic therapy in a murine model of oral candidosis. Oral Surgery, Oral Medicine, Oral Pathology, Oral Radiology and Endodontology, v. 109, n. 3, p. 392-401, 2010. Disponível em:< https://www.ncbi.nlm.nih.gov/pubmed/20060338>.

Acesso em: 25 ago. 2019. doi: 10.1016/j.tripleo.2009.10.006.

MIMA, E. G. O; PAVARINA, A. C; SILVA, M. M; RIBEIRO, D. G; VERGANI, C. E; KURASHI, C; BAGNATO, V. S. Denture stomatitis treated with photodynamic therapy: five cases. Oral Surgery, Oral Medicine, Oral Pathology, Oral Radiology and Endodontology, v. 12, p. 602-608, 2011. Disponível em: <https://www.sciencedirect.com/science/article/pii/S107 9210411003477>. Acesso em: 25 ago. 2019. https://doi.org/10.1016/j.tripleo.2011.05.019.

NÚÑEZ, S. C; RIBEIRO, M. S.; GARCEZ, A. S. PDT - Terapia fotodinâmica antimicrobiana na odontologia. 1 ed. Rio de Janeiro: Elsevier, 2013. $312 \mathrm{p}$.

NÚÑEZ, S. C; RIBEIRO, M. S.; GARCEZ, A. S. PDT - Terapia fotodinâmica antimicrobiana na odontologia. 2 ed. Rio de Janeiro: Elsevier, 2019. 253 p.

PEREIRA, C. A; DOMINGUES, N; SILVA, M. P; COSTA, A. C. B. P; JUNQUEIRA, J. C; JORGE, A. O. C. Photodynamic inactivation of virulence factors of Candida strains isolated from patients with denture stomatitis. Journal of Photochemistry and Photobiology B: Biology, v. 153, p. 8289, 2015. Disponível em: <https://www.sciencedirect.com/science/article/pii/S101 1134415002791>. Acesso em: 25 ago. 2019. https://doi.org/10.1016/j.jphotobiol.2015.08.029.

QUEIROGA, A. S; TRAJANO, V. N; LIMA, E. O; FERREITA, A. F. M; QUEIROGA, A. S; LIMEIRA Jr, F. A. In vitro photodynamic inactivation of Candida spp. by different doses of low power laser light. Photodiagnosis and Photodynamic Therapy, v. 8, n. 4, p. 332-336, 2011. Disponível em: < https://www.sciencedirect.com/science/article/abs/pii/s 1572100011004224?via\%3Dihub>. Acesso em: 05 dez. 2019.

QUEIROGA, A. S. Avaliação da atividade antimicrobiana da terapia Fotodinâmica sobre a inativação de espécies do gênero Candida: estudo in vitro. 2010. Tese (Doutorado em Odontologia) - Universidade Federal da Paraíba, João Pessoa, 2010.2 Disponível em: <https://repositorio.ufpb.br/jspui/handle/tede/6687>. 
Acesso em: 11 mar. 2019.

SCWINGEL, A. R; BARCESSAT, A. R; NÚÑEZ, S. C; RIBEIRO, M. S. Antimicrobial Photodynamic Therapy in the Treatment of Oral Candidiasis in HIV-Infected Patients. Photomedicine and Laser Surgery, v. 30, n. 8, p. 429-432, 2012. Disponível em: $<$

https://www.ncbi.nlm.nih.gov/pubmed/22730912>.

Acesso em: 25 ago. 2019. doi: 10.1089/pho.2012.3225.

SILVA, F. C; ROSA, L. P; PINHEIRO, A. L. B; KOGA-ITO, C. Y; ARAÚJO, B. P; VIANA, V, O; CAMPOS, L. A. N. P. Effectiveness of photodynamic therapy on Candida species isolated from oral samples of children exposed and not exposed to HIV. Revista Gaúcha de Odontologia, v. 64, n. 3, p. 271-279, 2016. Disponível em: <http://www.scielo.br/scielo.php?script=sci_arttext\&pid =\$1981-86372016000300271\&lng=en \&nrm=iso>. Acesso em: 07 set. 2019. http://dx.doi.org/10.1590/1981863720160003000052985 .

SNELL S. B; FOSTER, T. H; HAIDARIS, C. G. Miconazole Induces Fungistasis and Increases Killing of Candida albicans Subjected to Photodynamic Therapy. Photochemistry Photobiology, v. 88, n. 3, p. 596-603, 2012. Disponível em: $<$

https://onlinelibrary.wiley.com/doi/abs/10.1111/j.17511097.2011.01039.x>. Acesso em: 05 dez. 2019. doi:10.1111/j.1751-1097.2011.01039.x.

SOUSA, J. N. L; QUEIROGA, B. H; KOCERGINSKY, P. O; MARINHO, P. H. C; ARAKI, A. T. Photoinactivation of Candida albicans using methylene blue as photosensitizer. Revista Gaúcha Odontologia, v. 63, n. 4, p. 411-417, 2015. Disponível em <http://www.scielo.br/scielo.php?script=sci_arttext\&pid $=$ S1981-86372015010500411\&lng=pt\&nrm=iso $>$. Acesso em: 25 ago. 2019. http://dx.doi.org/10.1590/1981863720150003000063028 .

Submissão: 30/12/2019

Aprovado para publicação: $16 / 02 / 2020$ 